\title{
LOCALISED CHARGING EFFECTS INDUCED BY LOW VOLTAGE SEM OPERATION IN NON-CONDUCTIVE MATERIALS
}

\author{
Marion A. Stevens-Kalceff
}

School of Physics and Electron Microscope Unit, University of New South Wales, Sydney, NSW 2052, Australia.

Low voltage scanning electron microscopy (LVSEM) is defined for incident electron beam energies of $5 \mathrm{keV}$ or less. The reduction in the electron penetration depth improves spatial resolution for imaging and microanalysis. [1] For low incident electron beam energies, the secondary electron emission coefficient $\delta$, is enhanced in comparison with what is typically observed in the high energy limit. Thus low voltage operation may help to reduce charging effects in non-conductive materials. Irradiation induced trapped charge produces an electric field within the irradiated micro-volume of specimen which may influence the local structure. [2] This work investigates charging of insulating materials by low voltage electron beam irradiation in an SEM using advanced Scanning Probe Microscopy techniques.

Kelvin Probe Microscopy (KPM) or Scanning Surface Potential Microscopy (SSPM) is a specialized Atomic Force Microscopy technique in which long-range Coulomb forces between a conductive atomic force probe and a specimen enable the electrical potential at the specimen surface to be characterized with high spatial resolution. [3] A Veeco/ Digital Instruments extended Dimension 3000 Scanning Probe Microscope configured to operate in KPM/SSPM mode, has been used to characterize non-conductive materials irradiated in an SEM by electron beams of incident energies below $5 \mathrm{keV}$.

Significant localized residual charging is observed within the electron irradiated micro-volumes of non-conductive materials (e.g. see Fig. 1). Charge mitigation strategies in a conventional SEM include coating the specimen with a layer of thin grounded conductive material prior to imaging or microanlysis. Charging effects may be minimized in the low voltage operation limit for some non-conductive materials due to enhanced secondary electron emission, if suitable beam parameters have been chosen. The residual surface potential images resulting from low voltage electron irradiation are considerably different from those obtained from the same materials irradiated in the high energy regime (i.e. where $>5 \mathrm{keV}$ and $1-\eta>\delta$ where $\eta$ is the backscattered energy co-efficient. Net negative charging has been observed as expected [4]). The charging process in the low voltage limit is dynamic and dependent on a delicate balance between charging and secondary electron emission.

The reproducible characteristic surface potentials associated with the trapped charge have been measured using Kelvin Probe Microscopy and modelled using three dimensional conformal Finite Element Analysis [5] for comparison. This gives insight into the charging processes during low voltage SEM operation and the enables the resultant spatial distributions of the residual trapped charge to be estimated. The results of this work have implications for the low voltage microanalysis of non-conductive materials. 


\section{References}

[1] Joy, D.C. Joy, C.S. Micron 27 (1996) 247-263

[2] Cazaux, J. Journal of Applied Physics, 59(5), (1986) 1418-30

[3] Bonnell, D. A. Scanning Probe Microscopy and Spectroscopy: Theory, Techniques and Applications. Wiley-VCH. New York, 2001.

[4] Stevens-Kalceff M.A. Microscopy and Microanalysis (2003), submitted.

[5] Field Precision, Albuquerque, NM

[4] Support from the Australian Research Council and the Electron Microscope Unit at University of New South Wales is gratefully acknowledged.

Marion.Stevens-Kalceff@unsw.edu.au

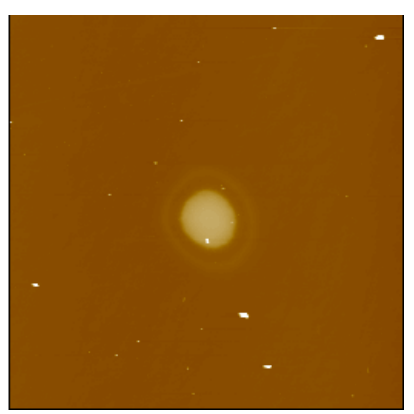

(a)

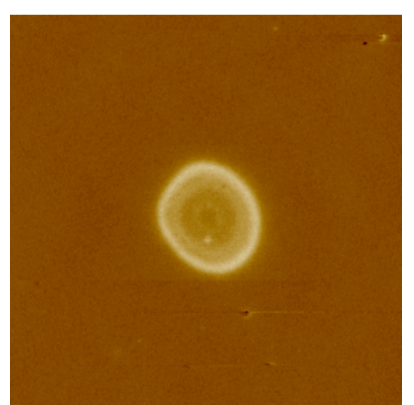

(b)

FIG. 1. 40x $40 \mu \mathrm{m}^{2}$ (a) surface topography and (b) KPM images of $\mathrm{SiO}_{2}$ irradiated with a defocused $4 \mathrm{keV}, 5 \mu \mathrm{m}$ diameter electron beam for $100 \mathrm{~s}$. The specimen was coated with a thin grounded layer of conductive carbon. This image shows significant residual surface potentials of $\sim 100 \mathrm{mV}$. 